\title{
Urgent Rescue of ‘Missing Rectus’ in Blowout Fracture
}

Hiroki Yano, Tomohiro Minagawa, Kana Masuda, Akiyoshi Hirano

Department of Plastic \& Reconstructive Surgery,

Nagasaki University School of Medicine, Nagasaki, Japan

\section{Address Correspondence to:}

\section{Hiroki Yano, M.D.}

Department of Plastic \& Reconstructive Surgery,

Nagasaki University School of Medicine,

1-7-1 Sakamoto, Nagasaki 852-8102, Japan

Fax: $\quad+81-95-849-7330$

Tel: $\quad+81-95-849-7327$

e-mail: h-yano@net.nagasaki-u.ac.jp 


\section{Summary}

Because guidelines for the treatment of blowout fractures have not been defined for urgent-care surgery, some patients retain sight-threatening strabismus after surgery. Authors presented a case involving immediate operation of blowout fracture based on CT findings and symptoms, demonstrating that early intervention may restore the full range of motion in the affected eye. The CT image showing the absence of the inferior rectus muscle on the orbital floor and no apparent fracture indicates the muscle strangulation. Immediate surgery must be performed to prevent irreversible muscular degeneration in such cases, rather than delaying the procedure several days.

\section{KEYWORDS}

blowout fracture, missing rectus, strangulation, vagal reflex, urgent operation 
There are no complete consensus on the use of surgical intervention in blowout fractures, although debate about the need for surgical correction or a temporal complication after injury has continued more than half a century. ${ }^{1-3)}$ The controversy may persist because many surgical practitioners, such as otolaryngologists, ophthalmologists, plastic and maxillofacial surgeons, have managed to correct orbital fractures. In fact, gaze restriction in some patients improves within several days under conservative management. ${ }^{4)}$ However, diplopia occasionally persists even after conservative therapy, and these patients later require surgical repair of the orbital floor as well as for the enophthalmus. ${ }^{5)}$ Furthermore, because of the degeneration of the extraocular muscle during conservative and/or delayed surgical approaches, some patients may ultimately have to undergo a strabismus operation because of gaze restriction. ${ }^{6)}$

In this report, we present a case of the blowout fracture that required an immediate operation based on the symptoms and CT imaging, which showed the absence of the inferior rectus muscle on the orbital floor 
and no apparent fracture indicating the muscle strangulation. In addition, we discuss the indication and the timing of surgical intervention.

\section{Case report}

An eight-year-old boy sustained facial injuries after falling from a horizontal bar, and presented diplopia along with severe nausea and vomiting. He initially visited a neurosurgeon and was referred to our department for a suspected blowout fracture. Although the CT image did not reveal an apparent fracture, it disclosed the absence of the inferior rectus muscle on the orbital floor (true 'missing rectus,' Fig. 1), possibly caused by herniation into the maxillary sinus. The patient's left eye movements - the upgaze and downgaze - were severely restricted (Fig. 2). No sensory loss was observed in the infraorbital nerve region. The diagnosis was a blowout fracture with a linear fracture of the orbital floor, and surgery was performed immediately to release the strangulated muscle.

Through an extended subciliary incision, the orbital periosteum was 
retracted to approach the orbital floor. As expected, a linear fracture with entrapment of the orbital content was confirmed. The herniated tissue was carefully restored after the floor was punched out with a fine osteotome, and then covered by grafting bone from the outer table of the calvarium. Two months after the operation, the diplopia was completely diminished in all directions (Figs. 3 and 4), and the patient was even able to play soccer.

\section{Discussion}

Although the target timing of the surgical intervention for the white-eyed blowout fracture has been to operate within several days of the event, ${ }^{78)}$ a true 'missing rectus' in blowout fracture of the orbit ${ }^{9)}$ requires more urgent intervention. In cases of the white-eyed fracture, the rectus muscle might have been entrapped rather than strangulated. In cases of entrapment without ischemic incarceration of the muscle and nerve, surgical treatment can be delayed several days, while a conservative 
approach is attempted. ${ }^{5,}$ 10) In the case of a true 'missing rectus,' the coronal CT reveals an absence of both the inferior rectus muscle above the floor and any apparent fracture of the floor; the result of this combination of factors is ischemia, which causes irreversible degeneration unless the ischemia is released by immediate surgery. ${ }^{11)}$

The mechanism underlying the missing rectus, based on hydraulic theory, ${ }^{12)}$ can be deduced. The orbital content including the muscle and the occulomotor nerve is forced out into the paranasal sinus through the flexible linear fracture that acts as a trapdoor. ${ }^{6)}$ Less dislocation of the fragment causes more serious strangulation in this type of blowout fracture. Furthermore, linear fracture, such as greenstick fractures in the long bones, are frequent in the pediatric population because of elasticity of immature bone. ${ }^{6,13,14)}$ Although recovery ability after injury is superior in children compared to adults, only immediate surgery can prevent sight-threatening strabismus in pediatric patients, before the resultant ischemia and inflammation lead to irreversible fibrosis and damage to the occulomotor 
nerve, and finally impair extraocular motility.

Before CT findings are obtained, symptoms can be indicative: Restricted downgaze may indicate muscular and nervous damage; restricted upgaze is a representative symptom of orbital floor fracture, i.e., the strangulation causes not only interruption of excursion at upgaze but also myogenic and neurogenic palsy ${ }^{15)}$ of the inferior rectus muscle itself. Other important signs of extraocular muscle damages include nausea and vomiting, ${ }^{13,14)}$ which are also common initial symptoms in brain injuries. Because stimulation of the extraocular muscle triggers the vagal reflex through the trigeminal nerve, also known as the oculocardiac reflex, injury to the muscle causes these symptoms. ${ }^{14)}$ Thus a patient with this type of blowout fracture generally exhibits nausea and vomiting and tends to close the affected eye because of severe diplopia and pain. When symptoms seem disproportionately severe for the injury, the vagal stimulation of trapped muscle must be considered. Because these symptoms depend on individual characteristics, a comprehensive decision regarding surgery must 
be indispensable and should include CT findings.

Burnstine drew up the indications for surgical repair in a blowout fracture. ${ }^{16)}$ We essentially agree with that algorithm but also emphasize that urgent repair is indispensable in cases of a true 'missing rectus'. Thus, we propose judging surgical indications based on etiology (Table 1). If the symptoms and CT findings reveal muscle strangulation, immediate surgical repair must be chosen. If the fracture type was punched-out or the muscle belly was confirmed on the floor, elective surgery could be selected through follow-up of the ocular movement for several days.

A patient suspected of blowout fracture who exhibits severe nausea and diplopia should immediately undergo CT examination, which is useful for judging the condition of the extraocular muscles. Helical CT can be performed on uncooperative or confused patients such as children, with minimum sedation or restraint, and multidirectional image slicing can be calculated from the raw data. ${ }^{1)}$ It is also convenient for ruling out intracranial injury. If the CT findings and symptoms reveal strangulation, 
surgical intervention must be immediately performed to rescue the 'missing rectus.' 


\section{REFERENCES}

1) Chandler DB, Rubin PA. Developments in the understanding and management of pediatric orbital fractures. Int Ophthalmol Clin. 2001; 41: 87-104.

2) Putterman AM, Stevens T, Urist MJ. Nonsurgical management of blow-out fractures of the orbital floor. Am J Ophthalmol. 1974; 77: 232-239.

3) Putterman AM. Management of blow out fractures of the orbital floor. III. the conservative approach. Surv Ophthalmol. 1991; 35: 292-298.

4) Nishida Y, Hayashi O, Miyake T, et al. Quantitative evaluation of ocular motility in blow-out fractures for selection of nonsurgically managed cases. Am $J$ Ophthalmol. 2004; 137: 777-779.

5) Egbert JE, May K, Kersten RC, Kulwin DR. Pediatric orbital floor fracture : direct extraocular muscle involvement. Ophthalmology 2000; 107: 1875-1879.

6) Grant JH 3rd, Patrinely JR, Weiss AH, Kierney PC, Gruss JS. Trapdoor fracture of the orbit in a pediatric population. Plast Reconstr Surg. 2002; 109: 482-489.

7) Jordan DR, Allen LH, White J, et al. Intervention within days for some orbital floor fractures: The white-eyed blowout. Ophthal Plast Reconstr Surg. 1998; 14 : 
379-390.

8) Tse R, Allen L, Matic D. The white-eyed medial blowout fracture. Plast Reconstr Surg. 2007; 119: 277-286.

9) Anda S, Elsas T, Harstad HK. The missing rectus: a CT observation from blow-out fracture of the orbital floor. $\quad$ J Comput Assist Tomogr. 1987; 11: 895-897.

10) Iliff N, Manson PN, Katz J, Rever L, Yaremchuk M. Mechanisms of extraocular muscle injury in orbital fractures. Plast Reconstr Surg. 1999; 103: 787-799.

11) Brady SM, McMann MA, Mazzoli RA, et al. The diagnosis and management of orbital blowout fractures: update 2001. Am J Emerg Med. 2001; 19: 147-154.

12) Smith B, Regan WF Jr. Blow-out fracture of the orbit: Mechanism and correlation of internal orbital fracture. Am J Ophthalmol. 1957; 44: 733-739.

13) Bansagi ZC, Meyer DR. Internal orbital fractures in the pediatric age group: characterization and management. Ophthalmology2000; 107: 829-836.

14) Cohen SM, Garrett CG. Pediatric orbital floor fractures: nausea/vomiting as signs of entrapment. Otolaryngol Head Neck Surg. 2003; 129: 43-47.

15) Kakizaki H, Zako M, Iwaki M, Mito $H$, Katori $N$. Incarceration of the inferior 
oblique muscle branch of the oculomotor nerve in two cases of orbital floor trapdoor fracture. Jpn J Ophthalmol. 2005; 49: 246-252.

16) Burnstine MA. Clinical recommendations for repair of isolated orbital floor fractures: an evidence-based analysis. Ophthalmology. 2002; 109:1207-1210 


\section{Figure Legends}

Fig. 1 CT image showing the 'missing rectus'. The inferior rectus muscle was absent on the orbital floor, and there was no apparent fracture. Note the well-imaged right inferior rectus muscle belly, compared with the left side. The orbital content, including the rectus muscle, herniated into the maxillary sinus through the invisible linear fracture.

Fig. 2 Range of eye motion before the operation. Downgaze and upgaze were greatly limited. Mydriasis arose because of medication administered for fundscopy.

Fig. 3 Range of eye motion two months after the operation. Eye movement was completely recovered, and the operative scar (the extended subciliary incision) was inconspicuous.

Fig. 4 Three-dimensional images reconstructed from the raw CT data $\theta f$ for this patient. Preoperation (above) and postoperation (below). 
Table 1

\begin{tabular}{|c|c|c|c|c|c|c|c|}
\hline \multicolumn{2}{|l|}{ Symptom } & \multicolumn{4}{|c|}{ CT findings } & \multirow{2}{*}{ Status } & \multirow{2}{*}{ Surgery } \\
\hline Impairment of ipsilateral gaze & Vagal relex & & Muscle involvement & Fracture type & Responsible tissue & & \\
\hline++ & ++ & \multirow{2}{*}{+} & true "missing rectus" & linear & \multirow{2}{*}{ muscle with fat } & strangulation & within hours \\
\hline+ & + & & "missing rectus" & punched-out & & incarceration & within days \\
\hline$+/-$ & $+/-$ & & - & either & fat (fibrous septa) & entrapment & within days if necessary \\
\hline
\end{tabular}


Fig. 1

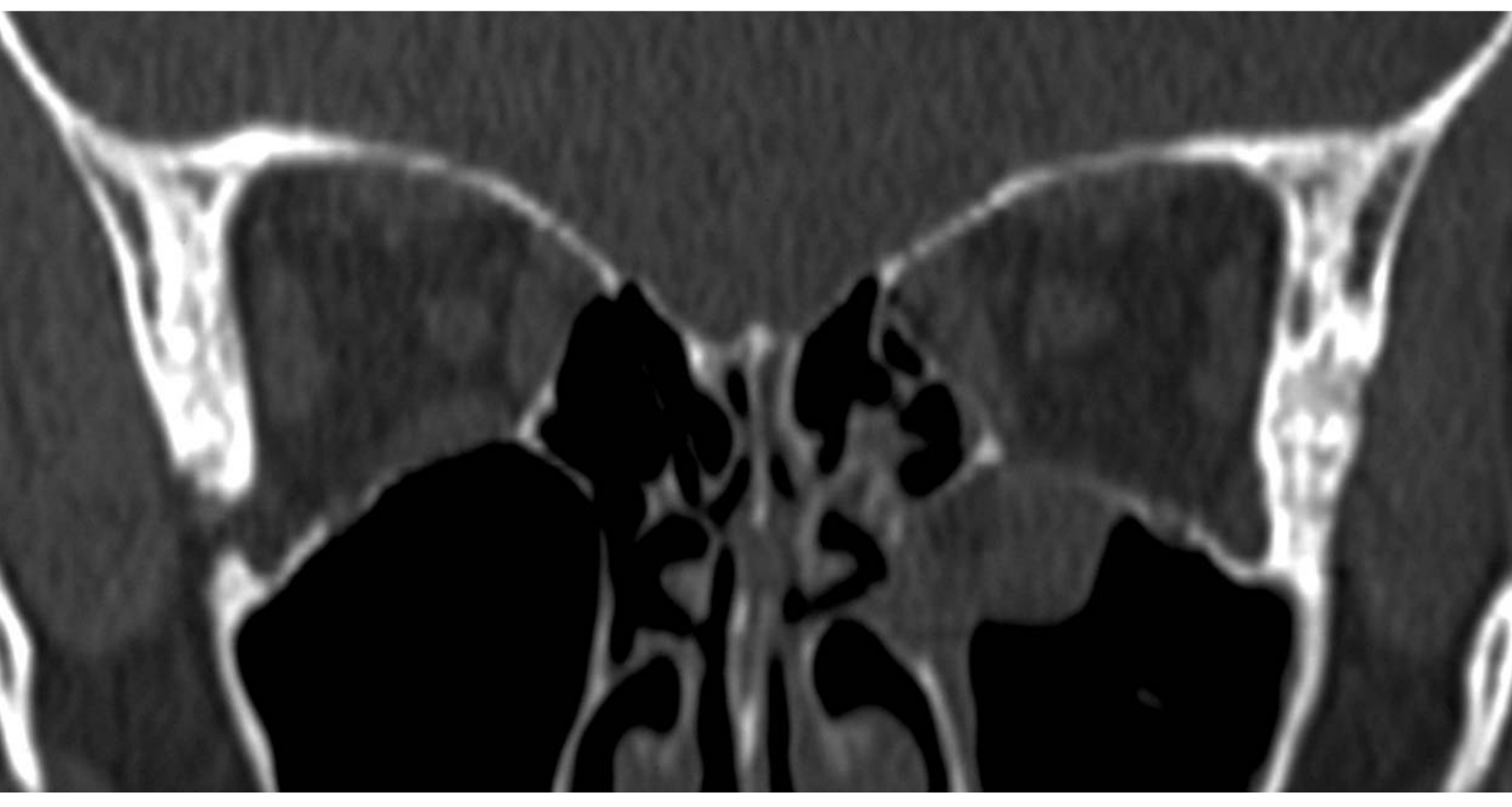


Fig. 2

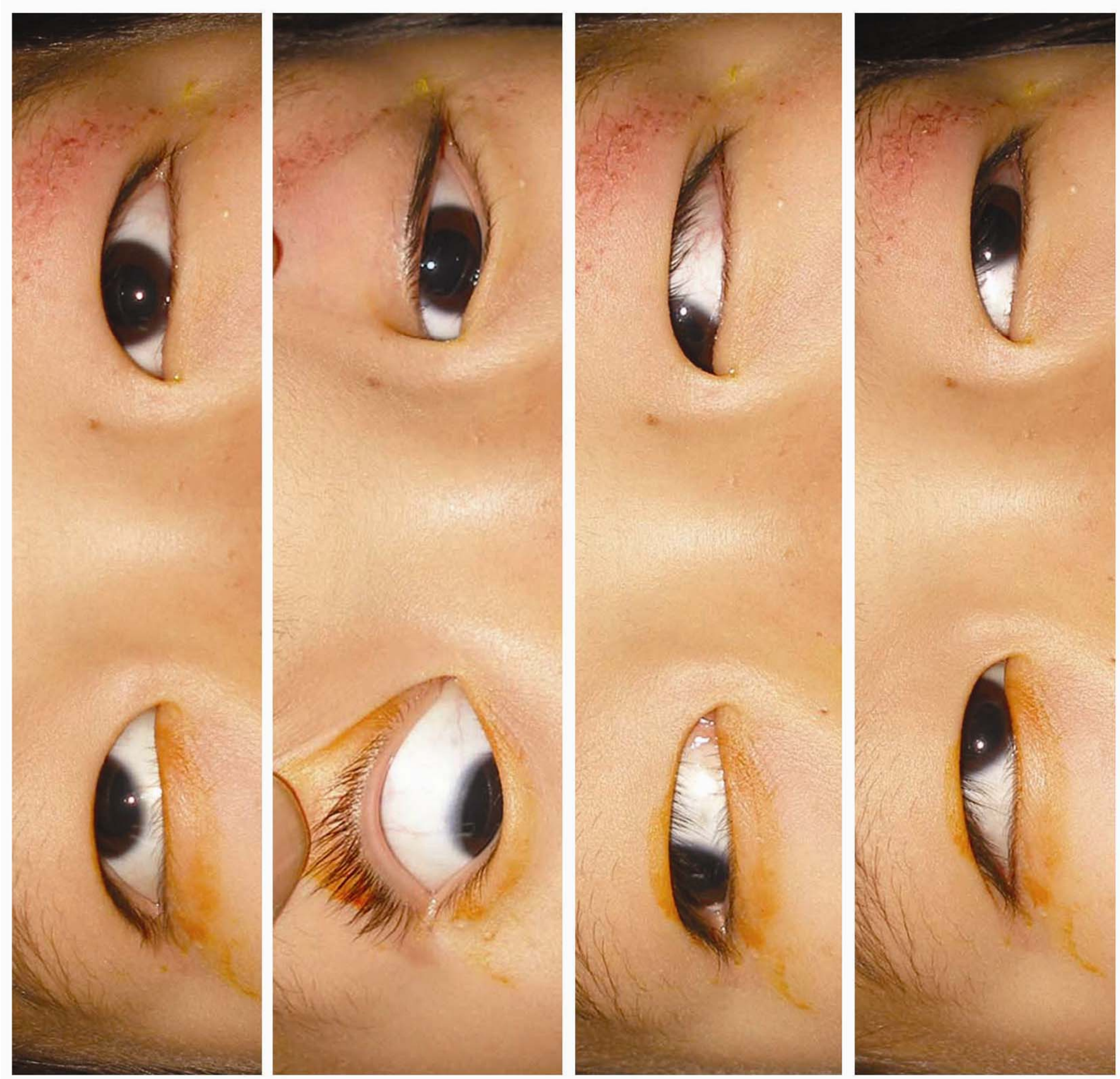


Fig. 3
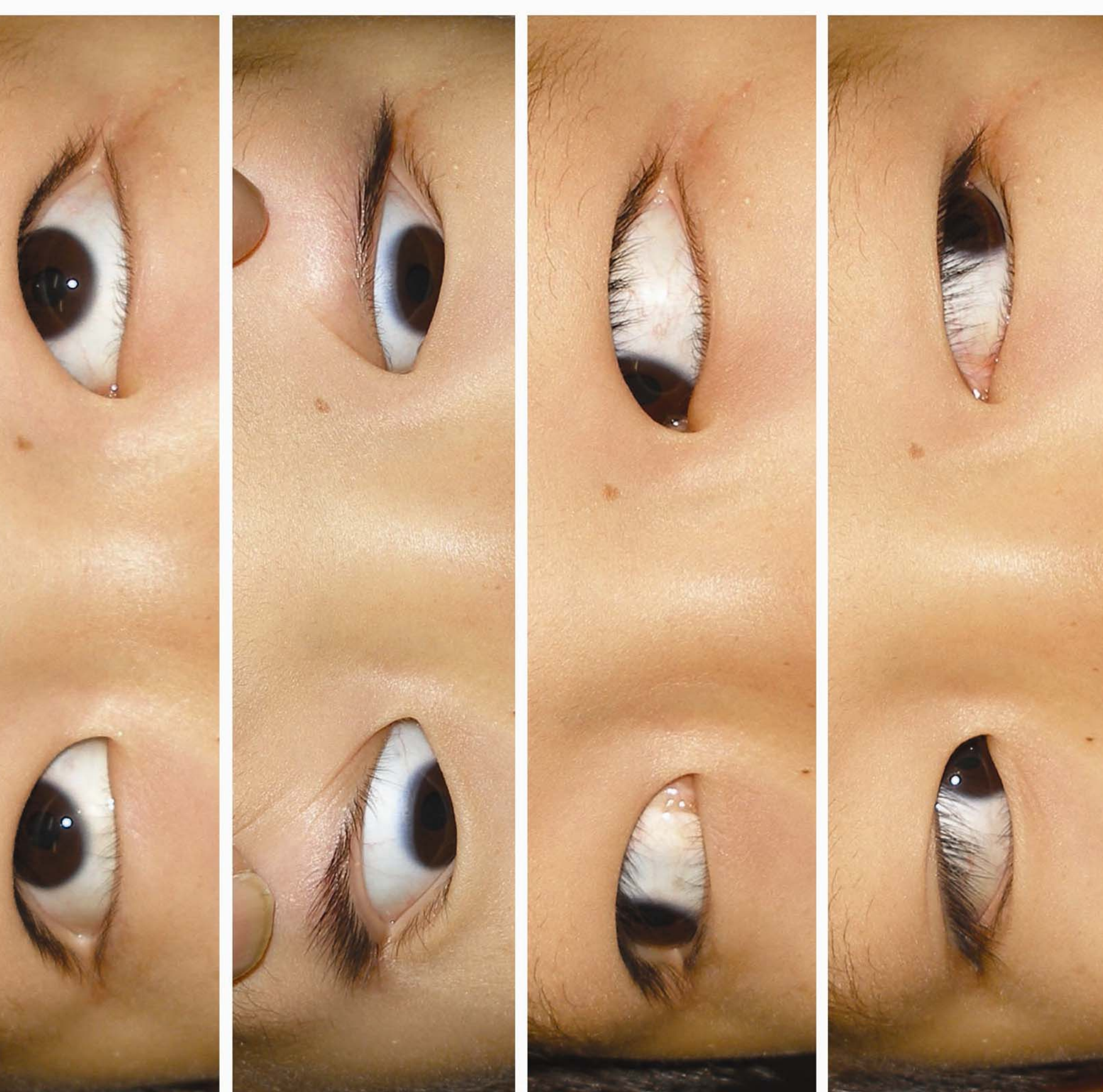

k 
